Original Research Paper

\title{
Using a Hierarchical Weibull model to Predict Failure Strength of Different Glass Edge Profiles
}

\author{
${ }^{1}$ David Thomas Kinsella and ${ }^{2}$ Johan Lindström \\ ${ }^{1}$ Construction Sciences, Lund University, Sweden \\ ${ }^{2}$ Centre for Mathematical Sciences, Lund University, Sweden
}

Article history

Received: 27-02-2020

Revised: 01-04-2020

Accepted: 30-04-2020

Corresponding Author

David Thomas Kinsella

Construction Sciences, Lund

University, Sweden

Email: david.kinsella@construction.lth.se

\begin{abstract}
The edge strength of glass is analyzed using a Weibull statistical framework based on 78 data samples from a range of experiments recorded in literature. Based on the analysis, a $45 \mathrm{MPa}$ strength value (computed as the lower bound in a one-sided confidence interval at the $75 \%$ level for the 5-percentile in the distribution) could be conservatively used with arrised, ground and polished edges when related to a reference length of $100 \mathrm{~mm}$ at an applied stress rate of $2 \mathrm{MPa} / \mathrm{s}$. The size effect can be represented by the usual weakest-link scaling formula with the Weibull modulus taken to be 8.0, 12.0, 8.0 and 6.5, respectively, for as-cut, arrised, ground and polished edges. It is estimated that static fatigue is best accounted for with a value of stress corrosion parameter about $n=16$. The results are obtained with random sampling $\mathrm{MC}$ in a hierarchical modelling approach with the Weibull parameters treated as nested random variables. By accounting for the influence of glass supplier as a mixed-effect in a linear statistical model, it is found that supplier effects are significant and important to consider along with others due to, e.g., stress rate and edge length exposed to maximum stress. The data samples which are limited to glass tested in an ambient environment using four-point bending fixture, show that Weibull statistics generally scatter considerably. Numerical investigations with random sampling show that shape parameter estimates scatter substantially when sample size is limited, which can explain some of the observed variability in shape more so for ground and polished edges than for as-cut and arrised. For the as-cut edge, it is suggested that the shape parameter is scale-dependent. The Weibull parameters are also estimated using a clustered likelihood estimator under the condition that the shape factor has constant value for each edge type.
\end{abstract}

Keywords: Glass, Edge, Strength, Statistics, Weibull, Hierarchical Modelling

\section{Introduction}

According to the latest European standard for glass in building, EN 16612:2019, there has not been a large-scale assessment of edge strength of the type undertaken for surface strength. From a practical perspective, it is conservative to assume that the edge is exposed to significant stress when a structure is subjected to the design load. For instance, even though maximum tensile stress occurs at the surface center point in a laterally supported plate subjected to uniform pressure, nevertheless, the corner edges are subject to considerable tension. Regarding other kinds of structural elements, such as beams and columns, it is evident that edge resistance cannot be neglected in the design process, in particular if edge resistance is deemed to be inferior to surface strength as standards would have it (EN 16612:2019, compare also DIN 18008-1:2010).
Experimental measurements of edge strength can be found in a wide range of journal articles, conference proceedings and academic dissertations which are available in the open literature (Table 1 gives a summary of the data used here). A comprehensive investigation into these results allow for an unprecedented analysis of glass edge strength within a Weibull statistical framework.

\section{Background}

\section{Manufacture}

The manufacture of float glass involves a long process line with production operations comprising a range of parameters of importance for the mechanical properties of the glass end-product, e.g., the thermal history which is carefully controlled to design the 
residual stresses (Le Bourhis, 2008; McLellan and Shand, 1984). The float process causes diffusion of tin into the surface that was in contact with the molten tin bath. This side is denoted the tin side whereas the opposite side is termed the air side (Krohn et al., 2002). The annealed glass is transferred to automatic cutting machines that produce standard size sheets with dimensions $6 \times 3.21 \mathrm{~m}^{2}$ (EN 572-1, 2012). When glass is cut it is first scribed under a sharp roller with oil applied in front of the cutting head to generate cracks which are subsequently driven through the thickness of the pane by flexuring the glass and breaking it in two. The result of the cut depends on the type of roller and the force, angle and velocity applied to it, in addition to the composition of cutting oil, all of which are controlled to generate median cracks and limit the introduction of lateral cracks, which degrade the edge quality, see also Müller-Braun et al. (2020) (Lawn, 1993; Le Bourhis, 2008). Moreover, the quality depends on the flexure stress and magnitude of surface residual stresses that may be present. In addition, the environment plays a role in the result (Le Bourhis, 2008).

By subjecting the as-cut edge to grinding operations, a range of edge profiles are produced. Here we consider the arrised, ground and polished types as illustrated schematically in Fig. 1. The arrising is performed with a cross-belt or cup wheel edging machine which introduce bevels at an angle of about $45^{\circ}$ to the surface. The result of arrising depends on the belt speed and direction for crossbelt machines and on the rotation speed for machines with cup wheels, as well as on the grinding pressure, the selected grain size and the order of application of grit sizes, in addition to the total age and usage of the machines (Kleuderlein et al., 2014; Veer, 2007). The optical quality of the ground edge is characterised by its roughness and smooth spots of as-cut glass may be present on the surface edge (site S23 in Fig. 1) depending on the amount of grinding performed (Vandebroek et al., 2014). The action of polishing is carried out in a similar manner to grinding, however, very little material is removed in the process (McLellan and Shand, 1984).

\section{Weibull Distribution}

The Weibull distribution (Weibull et al., 1952) for the strength $\sigma$ is:

$F(\sigma)=1-\exp \left(-\left(\frac{\sigma}{k}\right)^{m}\right), k>0, m>0, \sigma \geq 0$

where, $k$ and $m$ are scale and shape parameters. The corresponding density function is:

$$
f(k, m ; \sigma)=\frac{m}{k}\left(\frac{\sigma}{k}\right)^{m-1} \exp \left(-\left(\frac{\sigma}{k}\right)^{m}\right),
$$

and the coefficient of variation is:

$\operatorname{COV}=\sqrt{\frac{\Gamma \frac{(m+2)}{m}}{\Gamma\left(\frac{m+1}{m}\right)}-1}$,

where, $\Gamma(\cdot)$ is the Gamma function, i.e., $\Gamma(m)=(m-1)$ ! and generalized for non-integer $m$ (Rinne, 2009). For glass the Weibull distribution has been derived from assumptions regarding crack occurrences and sizes (see overview in Sec. Mathematical development of the Weibull model), providing links between the parameters in Equation (1) and the physical parameters of the glass; these links can be used when modelling glass strength.

Parameters of the Weibull distribution can be estimated using standard statistical theory, see Appendix A. To illustrate how the uncertainty in parameter estimates depends on the sample size, 50 point estimates of Weibull scale and shape parameters together with confidence bounds obtained from numerical simulations with random Weibull samples are shown in Fig. 2. The underlying scale and shape parameter values are $k=75$ and $m=10$.

\section{Mathematical Development of the Weibull Model}

The strength of glass is governed by the presence of flaws which turn into fracture sites when tensile stress reaches a critical level. The flaws are represented by cracks and the extension of a crack is modelled by an energy balance. Crack growth is prompted by either of three modes of deformation, viz. mode I, mode II and mode III (Irwin, 1958). Mode I refers to crack opening due to displacements normal to the crack plane surface. Mode II and III describe in-plane and out-of-plane shearing displacement cracking (Broek, 1983). As a simplification we consider only the impact of Mode I displacements. Failure is governed by the critical release rate of elastic strain energy. The mode I Stress Intensity Factor (SIF) for a sharp crack subjected to far-field tensile stress $\sigma$ acting perpendicular to the crack plane is:

$K_{I}=\sigma Y \sqrt{a \pi}$,

where, $a$ is the crack size and $Y$ is a geometrical configuration factor whose value in many cases is roughly equal to unity (Irwin, 1957; Hellan, 1984); e.g., for a straight-fronted planar edge crack $Y=1.12$ (Irwin, 1958). The fracture criterion is:

$K_{I} \geq K_{I c}$

where, $K_{I c}$ is the fracture toughness which for sodalime glass equals to about $0.75 \mathrm{MPa} \mathrm{m}^{1 / 2}$ (Mencik, 1992). 


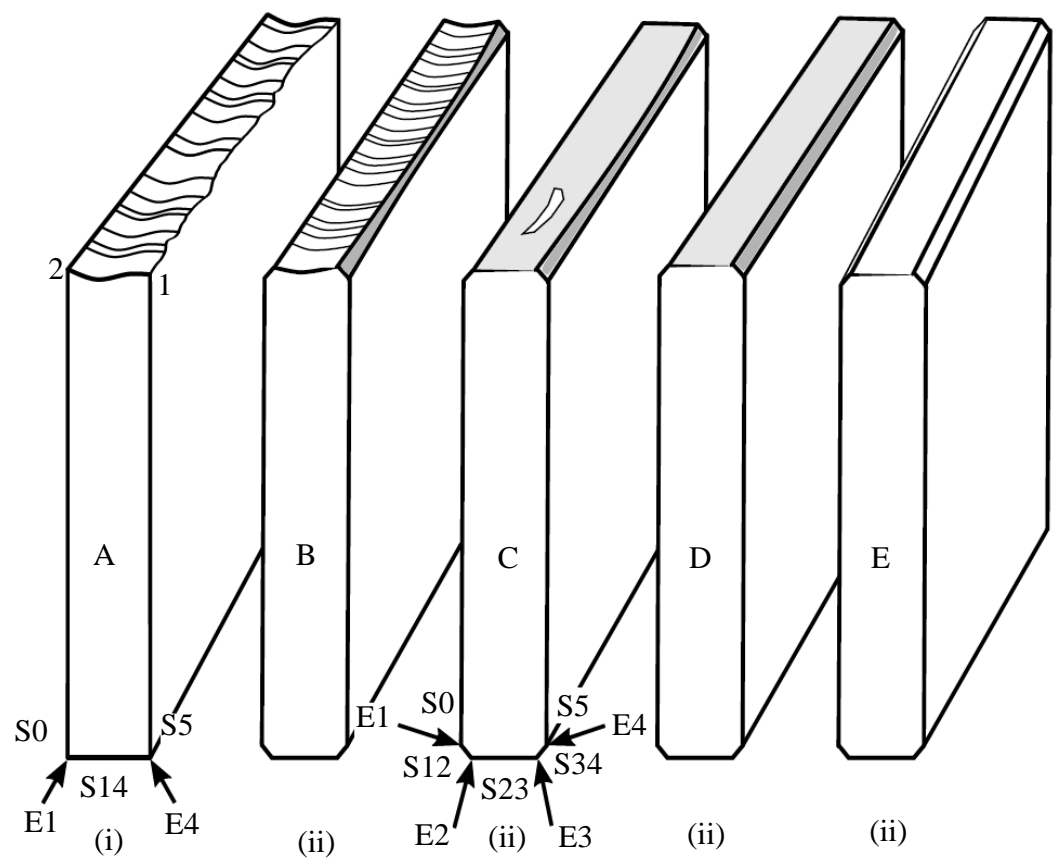

Fig. 1: Edge types. (A) As-cut with scored, 1 and non-scored, 2, edge. (B) Arrised. (C) Ground with smooth spots of as-cut glass remaining visible. (D) Ground. (E) Polished. Cross-sectional edge perimeter for (i) as-cut and (ii) arrised, ground and polished, respectively, is divided into zones where $S=$ surface, $E=$ edge
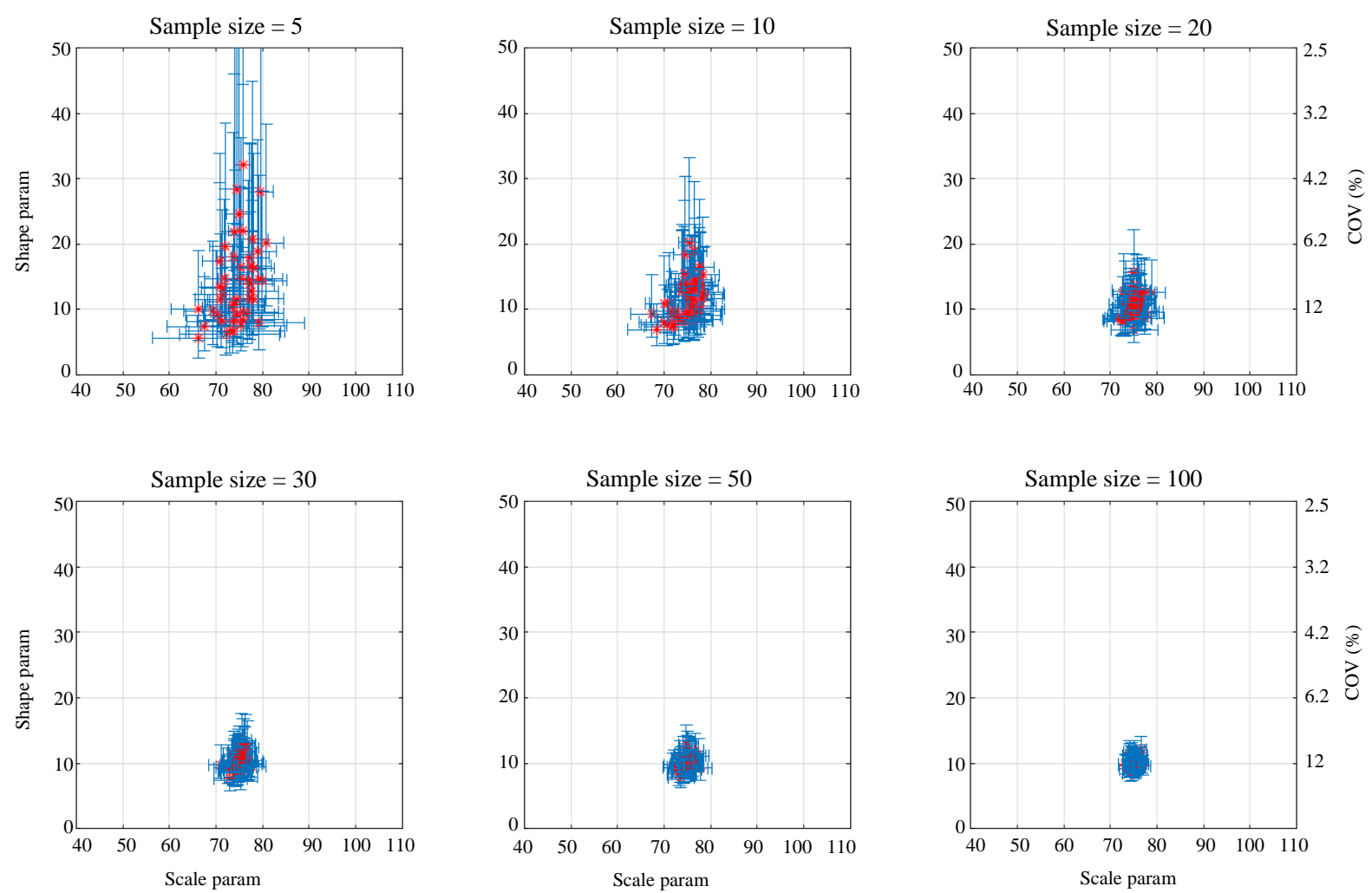

Fig. 2: Estimated confidence bounds for Weibull scale and shape parameters in numerical simulations of samples with varying size. True values: $k=75$ and $m=10$ 
Combining Equation (4) with (5) and assuming a homogeneous Poisson process for crack occurrence with Pareto distributed crack sizes, it can be shown (Mesarovic et al., 1992; Haldimann, 2006) that the total failure probability for an edge of length $L$ subjected to uniform stress is:

$$
P_{f}\left(L ; \sigma_{f}\right)=1-\exp \left[-\lambda_{0} \frac{L}{L_{0}} a_{0}^{r}\left(\frac{\sigma_{f} Y \sqrt{\pi}}{K_{I c}}\right)^{2 r}\right],
$$

where, $\lambda_{0} / L_{0}$ is the crack density, $a_{0}$ and $r$ are Pareto scale and shape parameters and the Pareto distribution is:

$$
F_{A}\left(a_{i}\right)=1-\left(\frac{a_{0}}{a_{i}}\right)^{r}, a_{0}>0, r>0 .
$$

While subjected to tensile stress in an atmosphere that contains water moisture, cracks in glass propagate subcritically due to stress corrosion (Charles, 1958a,b). For structural glass design considerations, subcritical crack growth is modelled using Equation (8) in which $v_{0}$ and $n$ are stress corrosion parameters and $K_{t h}$ is a threshold value of SIF below which crack growth arrest occurs, at approximately $0.20-0.27 \mathrm{MPa} \mathrm{m}^{1 / 2}$ (Evans, 1974; Haldimann, 2006):

$$
d a=\left\{\begin{array}{l}
v_{0}\left(\frac{K_{I}}{K_{I c}}\right)^{n} d t, K_{t h}<K_{I}<K_{I c}, \\
0, \quad 0 \leq K_{I} \leq K_{t h} .
\end{array}\right.
$$

Combining Equation (4) to (8), neglecting the crack growth arrest limit and assuming in addition a constant applied stress rate until failure, subcritical crack growth is accounted for in an approximation of the total failure probability as expressed in Equation (9) (Haldimann, 2006):

$$
P_{f}\left(L ; \sigma_{f}\right)=1-\exp \left[\begin{array}{l}
-\lambda_{0} \frac{L}{L_{0}} a_{0}^{r} \cdot \ldots \\
\ldots \cdot\left(\frac{1}{2} \cdot \frac{n-2}{n+1} \cdot \frac{v_{0}}{\dot{\sigma}}\right)^{\frac{2 r}{n-2}}\left(\frac{Y \sqrt{\pi}}{K_{I c}}\right)^{\frac{2 r n}{n-2}} \sigma_{f}^{\frac{2 r(n+1)}{n-2}}
\end{array}\right]
$$

Equation (9) is a good approximation in the case of low to moderate loading rates when $v_{0}$ is large enough (Haldimann, 2006).

Both Equation (6) and (9) can be written in the form of the Weibull distribution (1). For Equation (9), the corresponding scale and shape parameters are identified as:

$$
\begin{aligned}
& k=\left(\frac{1}{\lambda_{0}} \cdot \frac{L_{0}}{L}\right)^{\frac{1}{m}}\left(\frac{1}{a_{0}}\right)^{\frac{n-2}{2(n+1)}} \ldots \\
& \ldots \cdot\left(2 \cdot \frac{n+1}{n-2} \cdot \frac{\dot{\sigma}}{v_{0}}\right)^{\frac{1}{n+1}}\left(\frac{K_{I c}}{Y \sqrt{\pi}}\right)^{\frac{n}{n+1}} \\
& m=2 r \cdot \frac{n+1}{n-2}
\end{aligned}
$$

\section{Four-Point Bending Test Results Reviewed}

A schematic of the four-point bending arrangement is shown in Fig. 3. The bending strength is calculated as:

$$
\sigma=3 \frac{F l_{0}}{w d^{2}}
$$

where, $l_{0}$ is the distance between force and support and $d$ and $w$ refer to the cross-sectional width and height, respectively. Here, the beam is standing up on its edge and subjected to an in-plane configuration of bending. The maximum stress rate as function of deformation rate, $\dot{u}$, is given by:

$\dot{\sigma}=\dot{u} \frac{3 E d}{3 L-4 l_{0}}$

where, $E$ is Young's modulus equal to about $72 \mathrm{GPa}$ and $L$ is the distance between the supports (Mencik, 1992).

A total of 78 samples from four-point bending tests comprising some 1800 observations of edge strength were collected from literature and reviewed, see Table 1 for an overview. The data samples were obtained from experiments conducted on new annealed glass specimens broken in a four-point bending fixture in an ambient environment. No special coating was applied to the tension side of glass. The configuration of bending fixture was such that it took place in the plane of the specimen, i.e., with the glass beam standing up on its edge. The edge type was either as-cut, arrised, ground, or polished. The experiments were by all accounts consistently performed according to best practices and in many cases the procedure was guided by some standard document, e.g., EN 1288-3:2000. About 85\% of recorded data was observed using a stress rate lower than $3 \mathrm{MPa} / \mathrm{s}$. The maximum stress rate used in any of the experiments was below approx. $55 \mathrm{MPa} / \mathrm{s}$. In total 19 suppliers of glass were identified, however, where it was not verified in a few cases that those are not confounded with each other, as indicated in the table; the reason being that it was not verified that the glass tested in Veer et al. $(2006 ; 2009)$ and Veer and Rodichev (2011) and Vandebroek et al. (2012; 2014), respectively, were obtained from separate original batches even though it is likely so because experiment designs including specimen dimensions were different. 


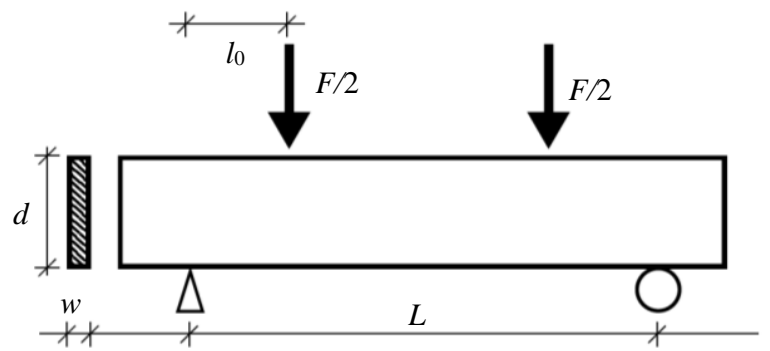

Fig. 3: Four-point bending test setup

According to one study on beams tested with an inplane configuration of bending, on average $20 \%$ and $13 \%$ of failures in as-cut and ground specimens, respectively, occurred from either site S0 or S5 in Fig. 1, i.e., from the main surfaces (Vandebroek et al., 2014). For the distribution of failure origins between sites $\mathrm{E} 1, \mathrm{~S} 14$ and E4, for the as-cut edge, Kleuderlein et al. (2014) found that some $92 \%$ occurred at the scored part of edge while in Vandebroek et al. (2014) it was $75 \%$. The statistics in Kleuderlein et al. (2014) and Vandebroek et al. (2014) show that failure origins in beams subjected to in-plane bending are distributed over the entire cross-sectional edge perimeter and extend even beyond into the main surface. In this context, we consider the surface to be defined by the pristine surfaces of glass that were in contact with the molten tin or air in the float process, whereas the edge is those parts of material subjected to various mechanical actions of scoring, cutting, arrising and grinding operations, etc. As a simplification, in this paper it is assumed that the in-plane bending configuration is a proxy for edge failures.

Ritter et al. (1984) reports the results from a wideranging testing programme on small annealed glass plates with ground edges subjected to four-point bending. A significant strength variability from laboratory to laboratory was noted and the estimated Weibull shape parameter for the edge population ranged from about 4.6 to 12.5 .

\section{Hierarchical Modelling of a Weibull Random Variable}

To model the 1782 observations we use a hierarchical approach with Weibull distributed errors:

$$
\sigma_{i j} \sim \operatorname{Weibull}\left(k_{i}, m_{i}\right)
$$

where, $\sigma_{i j}$ is the $j^{\text {th }}$ observation from the $i^{\text {th }}$ sample. Models for the resulting 78 shape and scale parameters, $k_{i}$ and $m_{i}$, are obtained by taking logarithms of Equation (10) and rewriting as:

$$
\begin{aligned}
& \log k=\frac{1}{m} \log \frac{L_{0}}{L}+\frac{1}{n+1} \log \frac{\dot{\sigma}}{\dot{\sigma}_{e q}}+\ldots \\
& \ldots+\frac{n-2}{2(n+1)}\left(\log \frac{1}{a_{0}}-\frac{\log \lambda_{0}}{r}\right)+\ldots \\
& \ldots+\frac{n}{n+1} \log \frac{K_{I c}}{Y \sqrt{\pi}}+\ldots \\
& \ldots+\frac{1}{n+1} \log \left(2 \cdot \frac{n+1}{n-2} \frac{\dot{\sigma}_{e q}}{v_{0}}\right),
\end{aligned}
$$

$\log m=\log \frac{n+1}{n-2}+\log (2 r)$.

Equation (14) is then used as a basis to formulate a statistical linear mixed effects model (McCulloch et al., 2008, Ch. 6) as follows:

$$
\begin{aligned}
& \log k_{i}-\frac{1}{m_{i}} \log \frac{L_{0}}{L_{i}}=\sum_{l=1}^{4} \beta_{l} \mathbb{I}_{\text {edgetypel }, i}+\ldots \\
& \ldots+\beta_{5} \log \frac{\dot{\sigma}_{i}}{\dot{\sigma}_{e q}}+\sum_{l=1}^{17} b_{l} \mathbb{I}_{\text {supplier }=l, i}+\varepsilon_{i}, \\
& \log m_{i}=\sum_{l=1}^{4} \gamma_{l} \mathbb{I}_{\text {edgetype }=l, i}+\ldots \\
& \ldots+\sum_{l=1}^{17} c_{l} \mathbb{I}_{\text {supplier }=l, i}+\varepsilon_{i}
\end{aligned}
$$

Here $\mathbb{I}$ are indicator functions for the categorical regressors; $L_{i}$ and $\dot{\sigma}_{i}$ are the recorded load span and stress rate in the $i^{\text {th }}$ sample; and we have chosen the reference load span and reference (or "equivalent") stress rate as $L_{0}=100 \mathrm{~mm}$ and $\dot{\sigma}_{e q}=2 \mathrm{MPa} s^{-1}$ respectively.

The model in Equation (15) is obtained from Equation (14) by the following steps and assumptions:

1. The first term on the Right-Hand Side (RHS) of Equation (14a), representing edge length (size) effect, has been included in the response variable on the LHS of Equation (15a)

2. The second term on the RHS of Equation (14a) represents the effect of the constant stress rate and depends on the stress corrosion parameter $n$. The reciprocal of $n+1$ is identified with the fixed-effect $\beta_{5}$ in Equation (15a)

3. Term number three on the RHS of Equation (14a) and the second term on the RHS of Equation (14b) correspond to the edge condition of glass. This depends on manufacturing processes (as outlined in Sec. Manufacture) and edge type, which governs the characteristics of flaw population, as modelled by $a_{0}, r$ and $\lambda_{0}$ in the Poisson process and Pareto distribution. The different edge types are considered as fixedeffects, given by $\beta_{l}$ and $\gamma_{l}$ for $l=1$ through 4 , while the supplier is a random effect captured in $b_{l}$ and $c_{l}$ 
4. Term number four on the RHS in Equation (14a) contains both a material parameter $K_{I c}$ and the fracture mechanics geometrical configuration factor $Y$ the latter of which is assumed to be approximately equal to unity. The effect of these are incorporated in the intercept value of the linear model

5. The last term on the RHS of Equation (14a) contains both stress corrosion parameters $n$ and $v_{0}$ of which the latter is assumed to be, on average, constant as long as the environmental conditions (temperature and $\mathrm{RH}$ ) are similar between experiments. Any random variability in $v_{0}$ will be captured in the error term $\epsilon_{i}$ and the dependence on $n$ is neglected as a simplification in the linear model

6. Finally, since $n \gg 1$, it is assumed that the ratios $n /(n$ $+1)$ and $(n-2) /(n+1)$ are approximately constant

The motivation for using a mixed effects model is that the effect of edge types and stress rate have physical interpretation, but differences between suppliers (and batches) are due to variability in the manufacturing process. Thus we are interested in the actual effect of edges and stress rate, while for the manufacturers we are more interested in categorizing the amount of variability among different manufacturers (see also Appendix B for a longer discussion).

Ideally the hierarchical model defined by Equation (13) and (15) should be estimated jointly, while accounting for the coupling in Equation (15). This could potentially be done using more advanced statistical algorithms such as Markov chain Monte Carlo and expectation maximization (Givens and Hoeting, 2013). However, to obtain a practically useful model we use a pragmatic two step approach where shape and scale for each sample are first estimated from Equation (13) and then used as response variables in two separate regressions in Equation (15) (Sampson et al., 2011, for a similar pragmatic approach to air pollution data.). In addition, a more involved model with mixed-effects for laboratory or with interactions between edge type and supplier would be desirable, but such a model is infeasible due to data sparsity; e.g., there are no cases in the published data where two (or more) laboratories uses the same batch of glass.

Given values of edge-type, stress rate and load span the model in Equation (15) can be used to predict shape and scale parameters; these parameters can then be used in Equation (13) to compute 5-percentile values. However, this would ignore the uncertainty due to different suppliers, a more correct model is obtained by integrating out (e.g., averaging over) the effect of different suppliers:

$$
\begin{aligned}
& f(\sigma)=\int f(\sigma \mid \operatorname{supp}) \cdot p(\operatorname{supp}) d \text { supp } \\
& \approx \frac{1}{N} \sum_{i=1}^{N} f\left(\sigma \mid k_{i}, m_{i}\right) .
\end{aligned}
$$

Here, $f(\sigma \mid$ supp $)$ is the Weibull distribution with parameters from Equation (15) given a known supplier and $p$ (supp) characterises the uncertainty in supplier effect, e.g., the variance of $b_{l}$ and $c_{l}$. The integral in Equation (16) is intractable and we use Monte Carlo integration to compute the distribution $f(\sigma)$ using the following steps:

1. Sample pairs of scale and shape parameters from Equation (15) while assuming that the glass could have been obtained from any supplier

2. Given scale and shape parameters the corresponding Weibull density, Equation (13), is computed

3. Steps 1 and 2 are repeated 10000 times and the results are averaged, providing a numerical approximation of the integral in Equation (16)

\section{Results}

The ordinary ML-estimates of the Weibull parameters, cf. Equation (19), for the data sets included in Table 1 are computed and illustrated in Fig. 4 where also the confidence bounds are shown. The results are grouped according to edge-type and load span dimension, additionally differences in supplier/batch (cf. Table 1) and edge thickness are indicated. For the as-cut and polished edge types additional samples with edge failures recorded in outof-plane configuration of four-point bending are included. These additional samples were obtained from Veer and Rodichev (2011), Muniz-Calvente et al. (2016) and Osnes et al. (2018) and were given supplier/batch indices 4, 22 and 23, respectively, cf. Table 1 and see also Table. 4.

Using Equation (15b), a LME model is applied and a constant shape factor per edge type is computed as fixedeffect, the value of which is provided in Table 2. The LME model of the shape factor is further illustrated in Fig. 5 which shows the computed fixed and random effects with approximate and simultaneous $95 \%$ confidence bounds.

Figure 6 illustrates the results for the scale factor when the LME model, Equation (15a), is fitted. In the middle is a subplot of the fixed-effects with approximate and simultaneous $95 \%$ confidence bounds included. In the bottom is a subplot of the fixed+random effects. The fixed-effect in Equation (15a) that corresponds to the reciprocal of $n+1$ is estimated at 0.0612 which produces a value of stress corrosion parameter at $n=15.3$ with approximate $95 \%$ confidence bounds [14.0, 16.9]. 

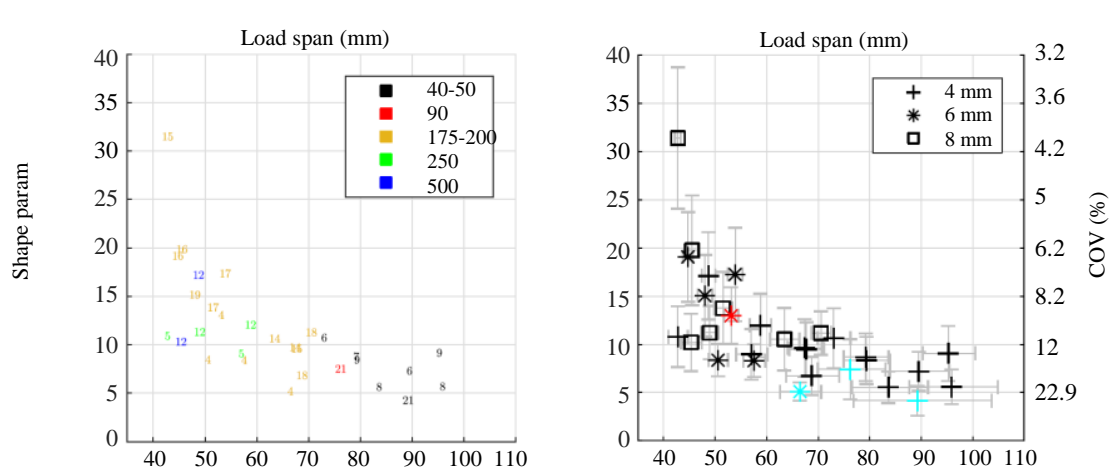

(a)
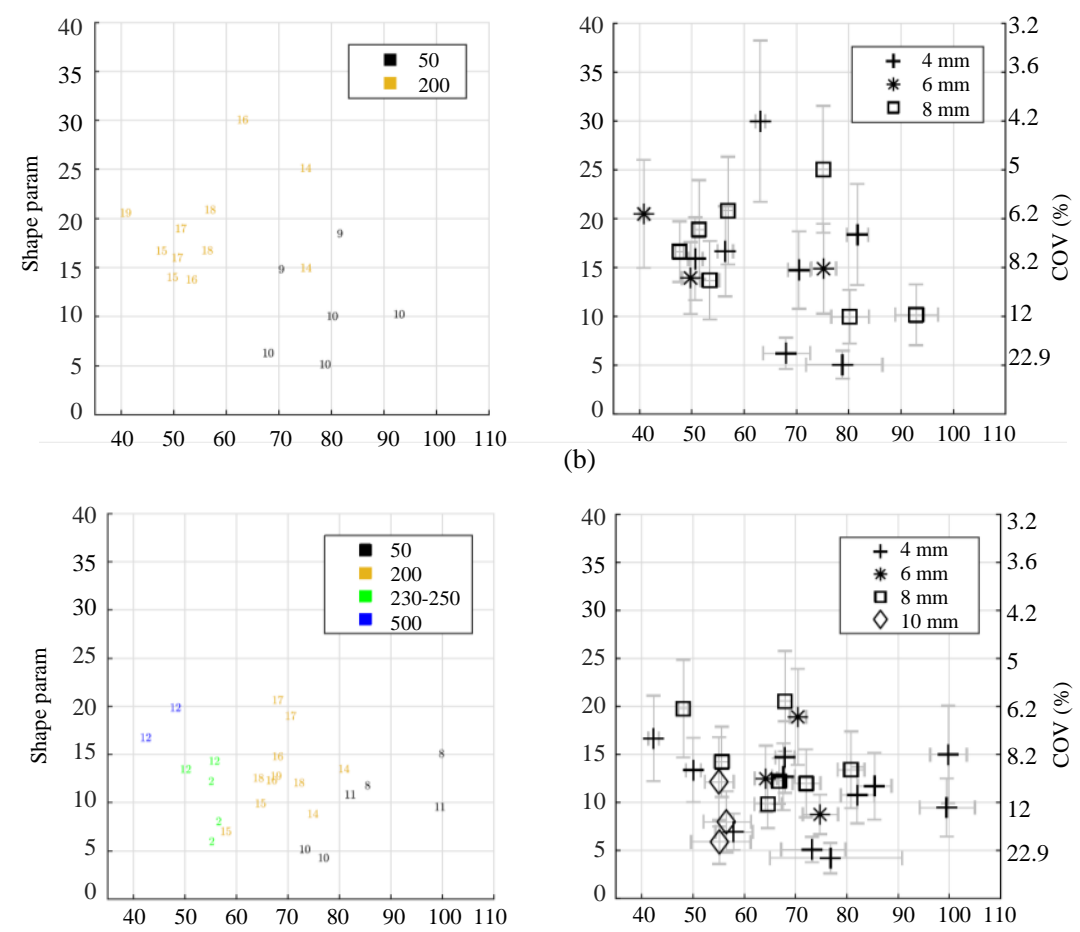

(c)
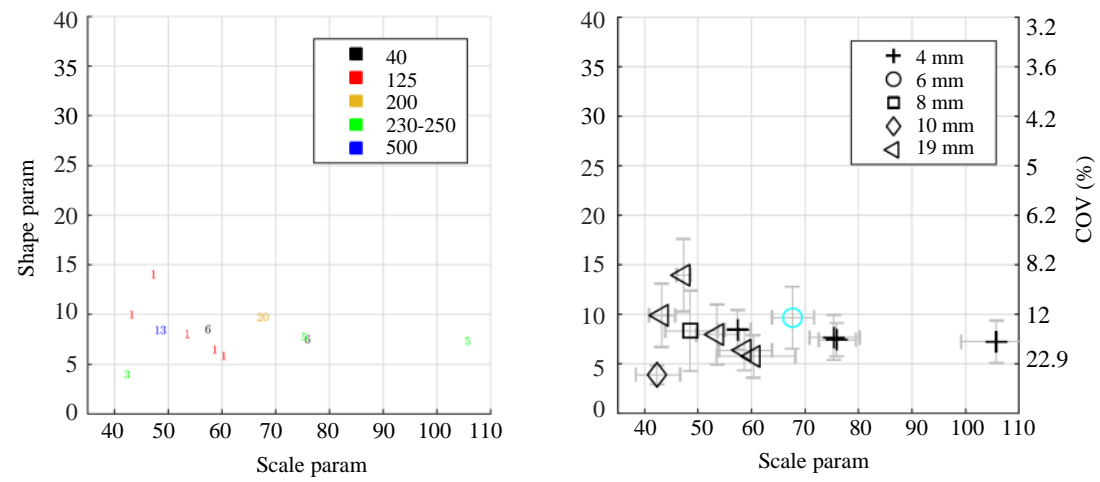

(d)

Fig. 4: Weibull statistics and sample data for the as-cut, arrised, ground and polished edge type in tests with in-plane bending configuration. Supplier/batch numbers are indicated on left-hand side and nominal edge thickness is indicated in symbolic marker,$+ \mathrm{o}$, *, etc., on right-hand side. For comparison additional points are added corresponding to fracture statistics with out-of-plane bending where the originally scored edge was in the tension (green marker) and compression (red marker) zone, respectively, as indicated in the right-hand subplots 

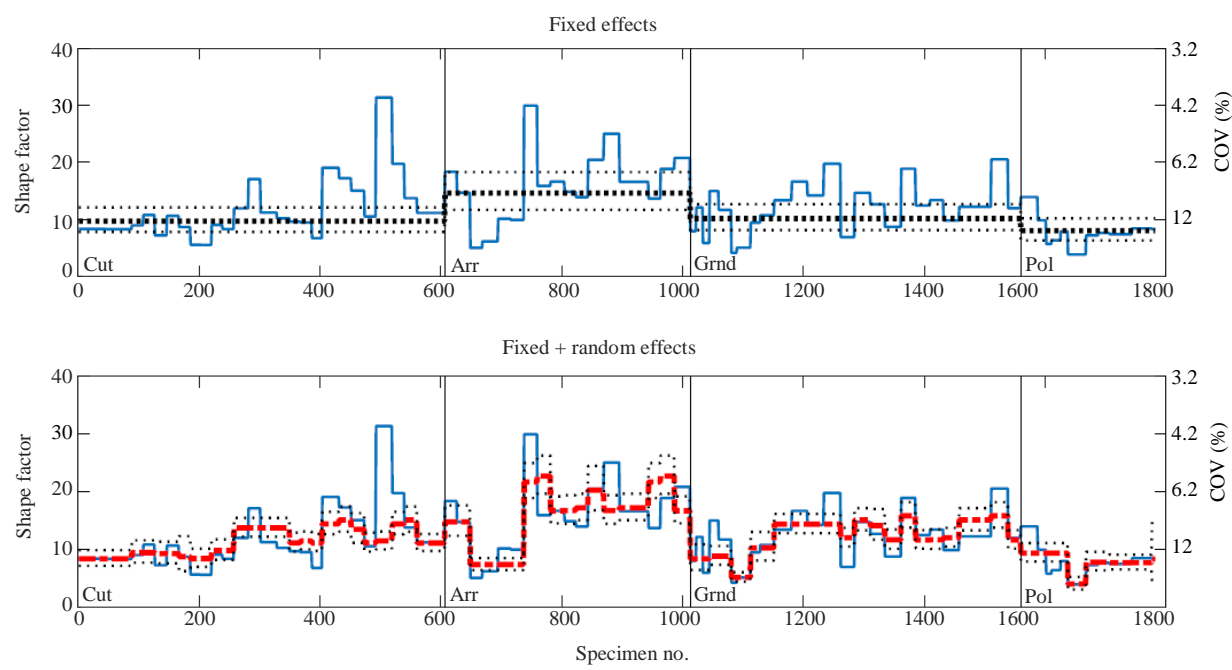

Fig. 5: Shape factor fixed and random effects according to LME model Equation (15b)
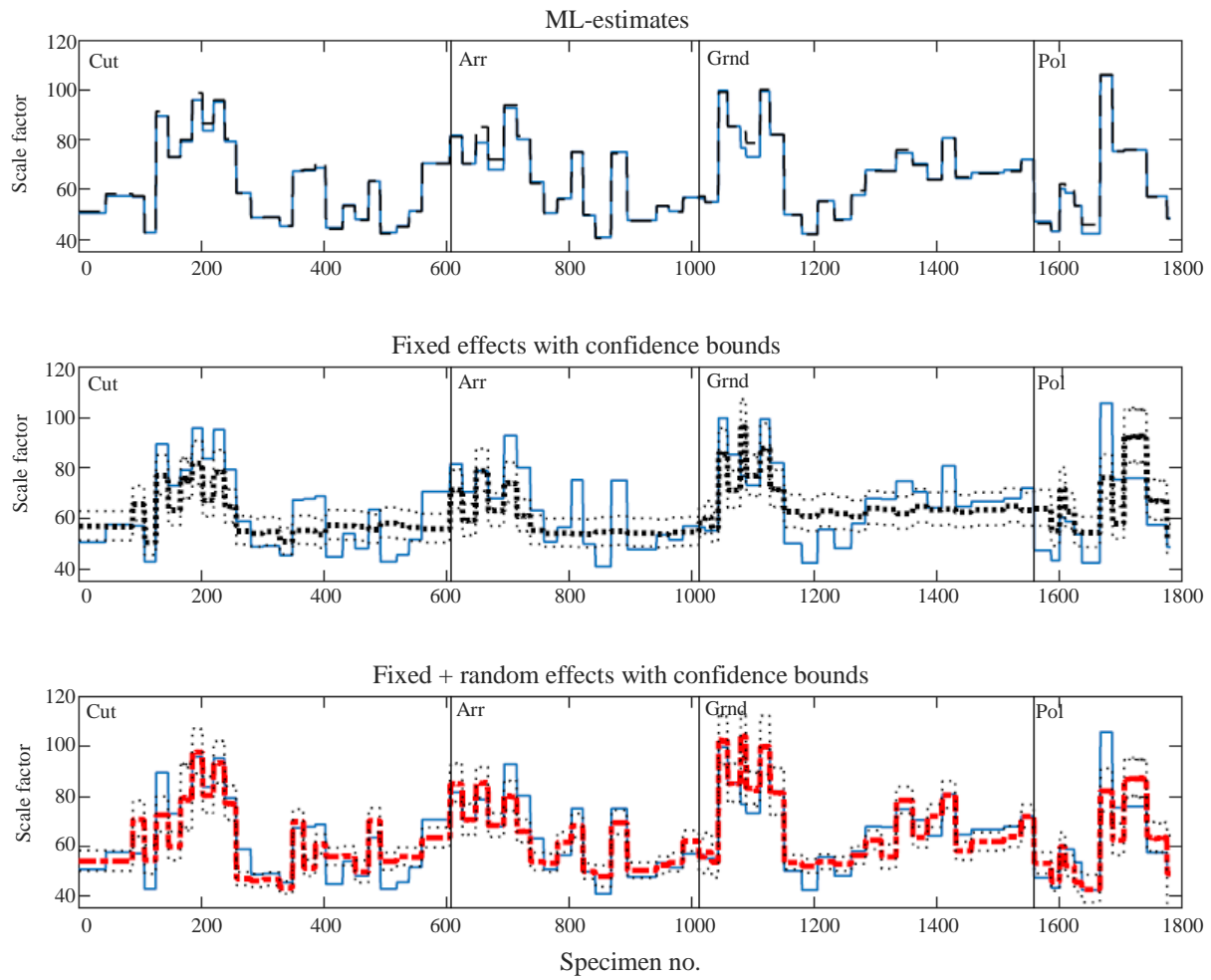

Fig. 6: Scale factor fixed and random effects according to LME model Equation (15a)

10,000 pairs of values of scale and shape parameter are drawn in random samples using the LME models, Equation (15b) and (15a), while assuming that the glass could have been obtained from either supplier (but with the same supplier, edge type, load span, etc., per each pair of parameters sampled). The stress rate is assumed to be $2 \mathrm{MPa} \mathrm{s}$. Since Equation (15a) contains the value of shape parameter, the shape factor is first simulated and then inserted into the model for scale parameter which is subsequently sampled. Then, median, 5\% fractile and 1 in 10,000 probability values of strength are computed in MC-simulations of Weibull distributions using the sampled pairs of random scale and shape parameters and the results are illustrated in Fig. 7 for each edge type and separated according to assumed load span dimension. The whiskers in the 
boxplots are extended beyond the quartile by one and a half times the interquartile range which implies about $99 \%$ coverage if the data are normally distributed. Also in these figures is indicated the lower bound of a one-sided confidence interval for the 5-percentile value at the approximate $75 \%$ confidence level. In addition, a mixture distribution function is computed, assuming that the glass could have been obtained from either supplier, in a MCsimulation based on the approximation formula in (16). Figure 8 shows the resulting mixture distributions per edge type and load span.

The proportion of variability in the response explained by the fitted models is given in Table 3 in addition to the fraction of the total unexplained variance that is explained by differences in supplier, $\sigma_{c}^{2} /\left(\sigma_{c}^{2}+\sigma_{\epsilon}^{2}\right) . \quad \sigma_{c}^{2}$ is the supplier variance, $\sigma_{\epsilon}^{2}$ the residual variance (see also App. B) and $\sigma_{c}^{2}+\sigma_{\epsilon}^{2}$ is the total variance not explained by fixed effects.
The LME model described so far results in the same shape factor for all data corresponding to the same edgetype. In the following, this is compared with the results from a clustered likelihood estimator, the clusters corresponding to each edge type, see also App. A. Given in Table 2 are the estimated shape factors calculated using Equation (20) and (15b). With Equation (20), the same edge-type has the same shape factor. The computation is based on the derivatives (21a) and (21b) and on the second derivatives (22a), (22b) and (22c) and is performed using a nonlinear minimization algorithm in MATLAB (MathWorks Inc., 2018). When the MLestimates are computed using either of Equation (19) and (20), i.e. with ordinary ML-estimation or alternatively with an estimation procedure that conditions the same edge-type to have the same shape factor, there is hardly any difference in resulting scale factor, as can be seen in the top subplot in Fig. 6 which compares these values.

Table 1: Batch/supplier index numbers corresponding to cited publication with samples of strength in in-plane conguration of four-point bending tests. Items marked with a star or dagger, respectively, refer to batches for which it was not verified that they are not confounded with each other. Edge finishing: $\mathrm{c}=$ as-cut, $a=$ arrised, $g=$ ground, $p=$ polished

\begin{tabular}{|c|c|c|c|c|}
\hline Publication & No. of samples & Total no. edge fail's & Batch index no. & Edge finishing \\
\hline Carre (1996) & 6 & 81 & 1 & $\mathrm{p}$ \\
\hline Veer et al. (2006) & 3 & 32 & $2^{*}$ & g \\
\hline Veer et al. (2009) & 1 & 30 & $3^{*}$ & $\mathrm{p}$ \\
\hline Veer and Rodichev (2011) & 2 & 83 & $4^{*}$ & $\mathrm{c}$ \\
\hline Vandebroek et al. (2012) & 4 & 77 & $5^{\dagger}$ & $\mathrm{cp}$ \\
\hline Lindqvist (2013) & 4 & 110 & 6 & $\mathrm{cp}$ \\
\hline$"$ & 1 & 19 & 7 & $\mathrm{c}$ \\
\hline$"$ & 4 & 73 & 8 & $\mathrm{cg}$ \\
\hline$"$ & 4 & 84 & 9 & ca \\
\hline$"$ & 6 & 101 & 10 & ag \\
\hline$"$ & 2 & 39 & 11 & g \\
\hline Vandebroek et al. (2014) & 8 & 202 & $12^{\dagger}$ & $\mathrm{cg}$ \\
\hline Kozlowski (2014) & 1 & 6 & 13 & $\mathrm{p}$ \\
\hline Kleuderlein et al. (2014) & 6 & 131 & 14 & cag \\
\hline$"$ & 6 & 163 & 15 & cag \\
\hline$"$ & 6 & 169 & 16 & cag \\
\hline$"$ & 6 & 138 & 17 & cag \\
\hline$"$ & 6 & 157 & 18 & cag \\
\hline$"$ & 3 & 74 & 19 & cag \\
\hline
\end{tabular}

Table 2: Estimated shape factor per edge type using Equation (20) or (15b) with approximate 95\% confidence bounds within parentheses

\begin{tabular}{lllll}
\multicolumn{1}{c}{ parentheses } & As-cut & Arrised & Ground & Polished \\
\hline ML-estimation procedure & 10.3 & 13.6 & 11.0 & 7.2 \\
LME modelxed-effect & $9.7(8.4,11.2)$ & $14.6(12.2,17.5)$ & $10.2(8.5,12.2)$ & $8.0(6.4,10.0)$ \\
\hline
\end{tabular}

Table 3: The proportion of variability in the response explained by the fitted model and the fraction of the total unexplained variance that is explained by differences in supplier

\begin{tabular}{llr}
\hline LME model & $\mathrm{R}^{2}$ & $\sigma_{c}^{2} /\left(\sigma_{c}^{2}+\sigma_{\epsilon}^{2}\right)$ \\
\hline Scale factor & 0.66 & 0.55 \\
Shape factor & 0.66 & 0.59 \\
\hline
\end{tabular}


Table 4: Data on the relative frequency of edge failures in laterally supported plates subjected to Uniform Pressure (ULP) and in Four-Point Bending (4PB) tests. For the ULP tests, only those edge failures are included that were unambiguosly identified as such. In some cases the experimenter recorded multiple potential fracture origins for a single specimen. In case of a mixture of potential surface and edge fracture sites for the same specimen, the associated observation was not included in the edge failure statistic. Hence, the statistic represents a lower bound on the relative frequency. OP $=$ out of-plane bending configuration

\begin{tabular}{lllll}
\hline Reference & Bend. type & Total no. fail's & No. edge fail's & Rel. freq. edge fail. \\
\hline Johar (1981) & ULP & 78 & 17 & 0.22 \\
Johar (1982) & ULP & 106 & 23 & 0.22 \\
Kanabolo and Norville (1985) & ULP & 206 & 54 & 0.26 \\
Calderone (1999) & ULP & 195 & 41 & 0.21 \\
Veer and Rodichev (2011) & 4PB OP & 89 & 84 & 0.94 \\
Muniz-Calvente et al. (2016) & 4PB OP & 30 & $14^{*}$ & 0.47 \\
Osnes et al. (2018) & 4PB OP & 93 & $21^{*}$ & 0.23 \\
Ritter et al. $(1984)$ & 4PB OP & $1263^{\dagger}$ & $1015^{\dagger}$ & $0.80^{\dagger}$ \\
\hline
\end{tabular}

*According to private correspondence

$\dagger$ Test conducted either in distilled water or in dry nitrogen gas
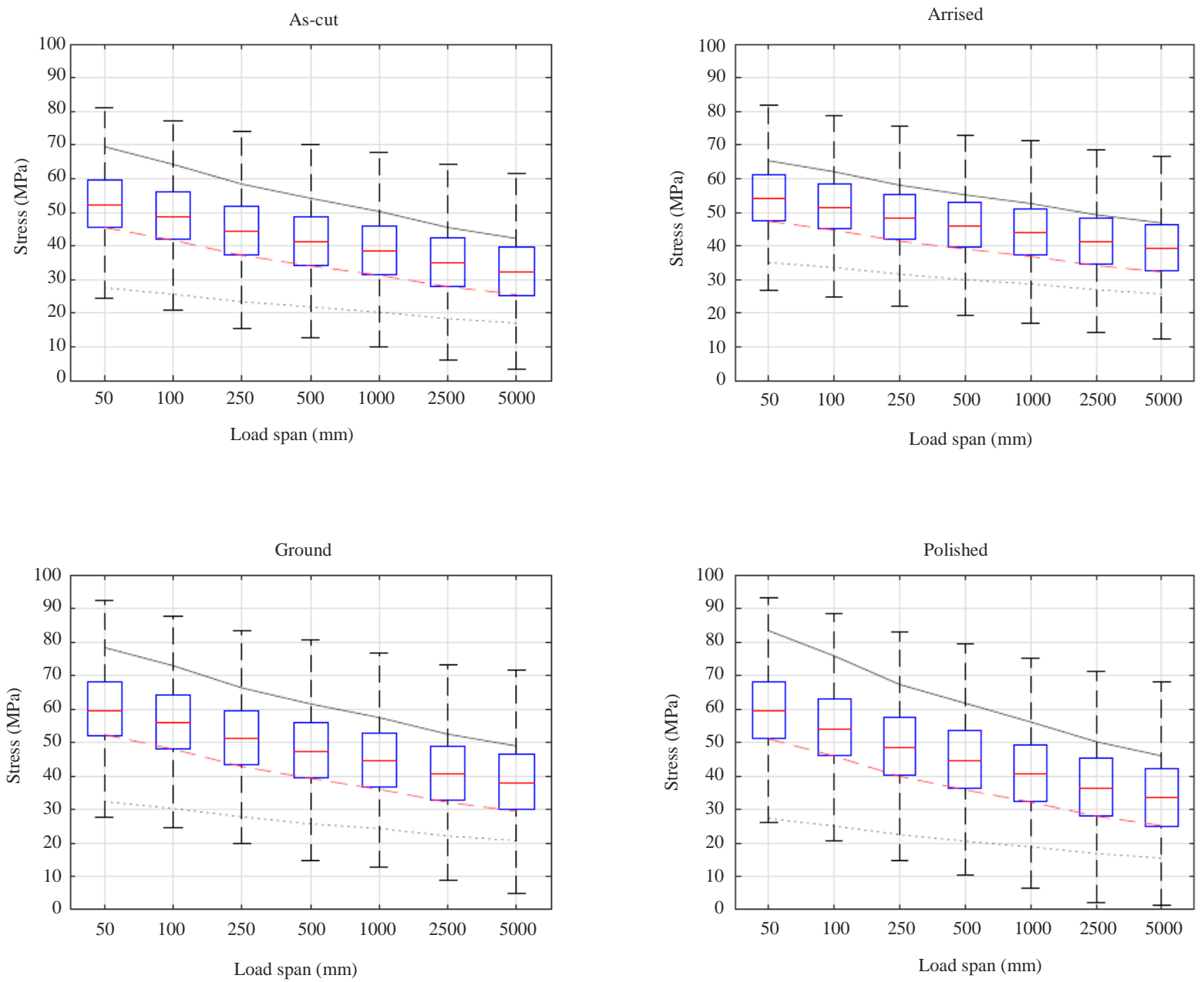

Fig. 7: Simulation of various strength percentiles per edge type at $2 \mathrm{MPa} / \mathrm{s}$ stress rate as function of load span dimension. The boxplots correspond with 5-percentile values. Dashed line is a curve fitted to the lower bound in a 75\% one-sided confidence interval for the 5-percentile. Solid line traces the estimated median strength (computed as median value of 10,000 point estimates per load span). Dotted line is the 1 in 10,000 probability failure stress (median value of point estimates) 

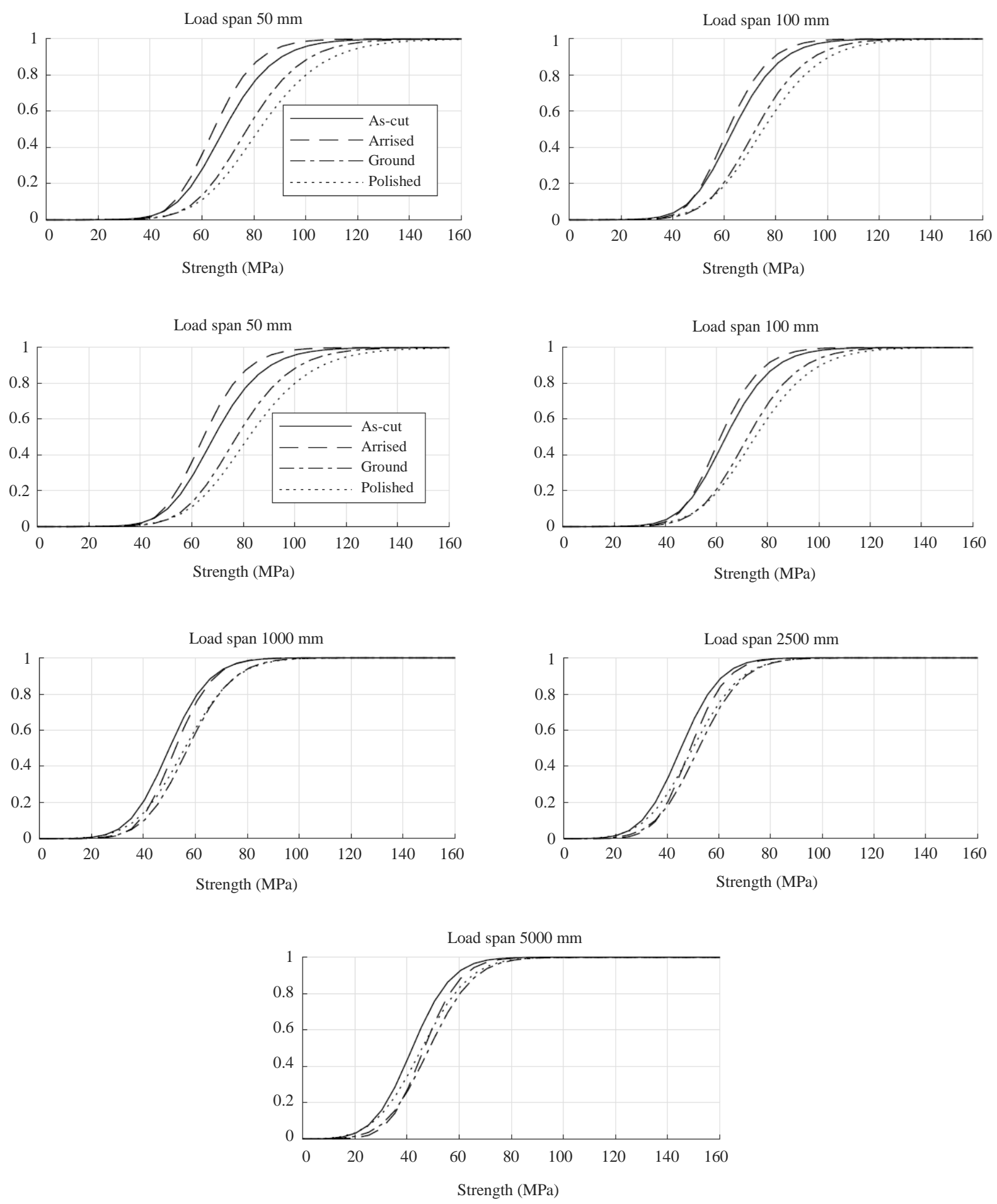

Fig. 8: Simulated mixture distributions for the strength at $2 \mathrm{MPa} / \mathrm{s}$ stress rate per edge type as function of load span

\section{Discussion}

The edge strength is important to consider in design of glass structures because in practice, the edge is prone to significant tensile stress even when maximum stress occurs away from the edge elsewhere on the surface. This is demonstrated in various experiments with foursided laterally supported plates subjected to uniform pressure for which the maximum tensile stress occurs at the centre point of the surface. According to a recent re- 
view of test results from hundreds of large laterally supported panes subjected to uniform pressure, it was found that none failed at the location of maximum principal tensile stress (Natividad et al., 2016). Moreover, according to a survey of almost 600 specimens tested with uniform lateral pressure, more than one in five failed at the edge, see Table 4. In addition, Table 4 shows that in four-point bending tests, on average more than half of failures originated from the edge when the configuration of bending was out-of-plane.

The additional mechanical action to the edge that occurs during production processes suggests that strength properties of the edge may not be equal to those of the pristine surface. According to EN 16612:2019, edge strength is generally considered to be inferior to surface strength and this is reflected in the prescription of an edge strength factor; the largest reduction is applied to the raw-cut edge and none at all to the polished edge. In a study involving eight participating laboratories (Ritter et al., 1984), it was found that for the results from seven out of eight laboratories the surface and edge failures were evenly mixed throughout the distribution so that a unimodal strength distribution often fitted the data well. However, it was also reported that with the results from one of eight laboratories, there was no intermixing of strength values from the edge and surface flaw populations and moreover, all of the surface failures were in the low strength portion of the distribution while edge failures were in the high strength portion. This clearly indicates that in some cases there can be significant differences in surface and edge strength distribution even as the specimens in this case (Ritter et al., 1984) were obtained from the same supplier and were randomized prior to being delivered to each of the participating laboratories. Moreover, these results apparently contradict the concept in EN 16612:2019 where the edge strength is deemed to be inferior to surface strength. Evidently, more research is needed to verify if the edge reduction factors adopted in the standards are correct. Figure 8 indicates that polished edges are generally the strongest.

Comparing different edge types with each other, see also Fig. 4, the polished edge corresponds with samples of either a similar or a larger coefficient of variation, on average, than other edge types. The optical appearance of the polished edge is superior, however, predictionmaking of its strength value is potentially associated with greater uncertainty than for other finishings. The selected values for edge strength factor in EN 16612: 2019 could be non-conservative.

The edge strength is complex to model due to the range of factors that can have a confounding effect. Even when the number of factors are limited by restricting the analysis to e.g., a fixed load span for a specific edge type at a specified rate of applied stress in a controlled environment, it is seen that strength values scatter substantially and it is concluded that the variation cannot be accounted for by a single standard statistical distribution, compare also Veer (2007). Rather, samples are fitted by Weibull distributions with scale and shape parameters that exhibit random variability in addition to systematic effects according to e.g., weakest-link scaling.

Considering the recorded data results in a Weibull statistical framework, it appears that a major confounding factor is related to manufacturing and production line processes which can be assumed to vary with location and over time due to e.g., variability in grinding wheel settings and machine properties, presence of residual compressive stresses and variations in environmental conditions during manufacture to the extent that it may affect the end-product (Veer, 2007; Kleuderlein et al., 2014; Le Bourhis, 2008). Table 3 shows that about two thirds of the variability in the LME models fitted to the Weibull scale and shape factors is explained by physical interpretation (edge-type, stress rate, weakest-link scaling). Of the remaining variability, between $55 \%-60 \%$ is related to supplier or batch effects. Approx. $14 \%$ of total variability is not explained by the model and can be attributed to, e.g., local effects during testing of each individual specimen and sample-to-sample effects including inter-laboratory differences, presence of residual compressive stresses and of course errors in the treatment of stress corrosion with the approximate theory applied.

The numerical investigation into sample size effect on computed Weibull parameter estimates, see Fig. 2, shows clearly that when sample size is limited, the shape parameter estimate scatters substantially to produce what might appear like a statistical artefact. This might explain most of the variability in observed shape parameter estimates from recorded test results for ground and polished edges (cf. Fig. 4c and 4d). It corresponds to the conclusion drawn in a previous large scale investigation of the strength of small glass plates with ground edges according to Ritter et al. (1984), where it was noted that whereas "the variability observed in the Weibull slope parameter, $m$, was close to that expected from the statistical reproducibility of the strength test [...] the variability in the median strength from laboratory to laboratory was much greater than that due only to statistical considerations." For the arrised and in particular as-cut edge (Fig. 4a and 4b), however, variability in shape parameter is hardly within statistical reproducibility.

The environmental conditions are known to significantly affect the observed strength due to stress corrosion (Charles, 1958b; Brown, 1974); at higher rates of applied loading, the strength is increased. In the present analysis, it was assumed that the environmental conditions during testing were approximately identical. This is a simplifying assumption however, as the recorded values of temperature and relative humidity in the experiments ranged from $15^{\circ} \mathrm{C}$ to $25^{\circ} \mathrm{C}$ and from 
about $23 \%$ to $70 \%$, respectively. According to a survey of measurements of stress corrosion parameter $v_{0}$ in Haldimann (2006) and Schula (2015), its value was found to vary substantially even under seemingly identical environmental conditions. This points to a potentially confounding effect on the estimation of scale factor in the present study and it is expected to contribute to the error terms in the LME model (15a). In addition, while applying Equation (9), the existence of the threshold limit value $K_{\text {Ith }}$ is ignored, however, this is assumed to have a limited effect on the results.

Although a general size effect is evident when considering Fig. 4, due to the scatter of individual statistics it is hard to verify that a proper assessment of it was made when based on a few sample statistics alone. The variation in estimated scale parameter for an identical load span at the same loading rate can be considerably large as the results for the as-cut and arrised edge type demonstrate. In conclusion it is recommended that size effect for glass edge be addressed in wide-ranging studies with a diversity in glass supplier, thickness, etc., perhaps also including diversity in participating laboratories. According to results from MC-simulations, see Fig. 7, the size effect can be expressed in terms of the value of 5-percentile strength with the ordinary weakest-link scaling formula, Equation (17), i.e., the decrease in strength from $\sigma_{1}$ to $\sigma_{2}$ as the load span increases from $L_{1}$ to $L_{2}$ is approximated well by the usual formula:

$$
\sigma_{2}=\sigma_{1}\left(\frac{L_{1}}{L_{2}}\right)^{\frac{1}{m}}
$$

if $m$ is taken as 8.0, 12.0, 8.0 and 6.5, respectively, for the as-cut, arrised, ground and polished edge types. These values are close to those given in Table. 2 . The corresponding fit is illustrated in Fig. 7 with a dashed red line where scaling is performed from a baseline strength value at load span $100 \mathrm{~mm}$. The load spans represented in the experiment data range from about $50 \mathrm{~mm}$ to $500 \mathrm{~mm}$. In practical circumstances when glass is used in structures, the effective load span can be larger than so and more research is needed to verify how the size effect is manifest and properly extrapolated. Figure 4 also shows the 1 in 10,000 probability failure stress with a dotted line which lies above $20 \mathrm{MPa}$ for all load spans that are represented in the data that was used to fit the model.

The characteristic 5-percentile strength values that were estimated in the present study, see Fig. 7, suggest that the $45 \mathrm{MPa}$ value for glass strength that is mentioned in EN 572-1:2012 (and which is further discussed in an annex to EN 16612:2019) could be used for the edge if it is computed as the lower bound in a one-sided confidence interval at the $75 \%$ level and, if it is related to a reference length of $100 \mathrm{~mm}$ at an applied stress rate of $2 \mathrm{MPa} \mathrm{s}^{-1}$ except for the as-cut edge which warrants a reduction of about $5 \%$ and the ground edge which allows for a increase of $5 \%$. The size scaling would then follow from Equation (17). However, the difference in estimated strength between edge types is not large and a simplification could be made. So long as the as-cut edge is not considered, the characteristic values for the polished edge in Fig. 7 could be adopted conservatively for either of the arrised, ground and polished edge types.

In the present study, the edge size was measured by its length, however, weakest-link scaling could be considered in terms of edge area rather than edge length so that if $w_{0}$ denotes a reference thickness then:

$\log k \propto \frac{1}{m} \log \frac{L_{0} \cdot w_{0}}{L \cdot w}$

where, $L_{0} w_{0}$ is the reference area and $L w$ is the given edge area. With increasing thickness, edge size is greater and in consequence, the probability for the edge to contain a severe flaw increases according to classical weakest-link scaling argument. Or, another measure might be used, such as a representative volume that extends into part of the main surfaces (zone S0 and S5 in Fig. 1) in which case edge size would no longer be a simple product of thickness times length. However, increasing thickness is associated with differently chosen cutting angles in the production method. Therefore, it can be assumed that flaw population characteristics vary with thickness (Lindqvist, 2013; Veer, 2007). For such reason, glass thickness may interact with edge length and edge type to produce an effect on strength in ways that may not be self-evident. Consequently, Equation (18), although logical from the point of view of classical weakest link scaling principles, does not sufficiently express the effect on strength due to edge thickness variations. This was noted in the present study when various adaptations of the LME in (15a) and (15b) were investigated and compared using the AIC information, see App. A. For the shape parameter, it was seen that supplier/batch, edge type and thickness where the most important factors (in the given order) to consider in search of better performing models. For the scale parameter, slightly better performing models where produced when edge thickness was included as a fixedeffect rather than accounted for by using Equation (18) and, in fact, the same was seen for load span length, too. This indicates that weakest-link scaling of strength due to edge size is not entirely a simple matter of scaling according to the formula in Equation (18). Instead, it is suggested that edge characteristics in terms of flaw population vary with thickness and even with length. More research is needed to verify the effect on strength due to edge thickness and to better understand the proper way to measure edge size for weakest-link scaling purposes. 
According to Veer and Rodichev (2011), lateral cracks generated by scribing are located at a greater depth than the initial surface microcracks. The length of lateral cracks is also greater than the width of the score itself. The hypothesis laid forward in Veer and Rodichev (2011) is that some of the lateral cracks are so large and are located at such depth that they remain partially or in full even after grinding and polishing operations. Others, however, appear to disagree. In Vandebroek et al. (2014) it is said that "[a]fter grinding, one can assume that the damage caused by cutting has vanished." And in Kleuderlein et al. (2014) it is suggested that "damages in the form of microcracks caused by the cutting process are eliminated or at least significantly reduced by arrising or grinding." On a similar note, Sglavo et al. (2007) who performed three-point bending tests on monolithic glass beams with an out-of-plane configuration of the bending, reported that the position of the originally scored edge, i.e., in the tension zone or in the compression zone, had an insignificant effect on the strength when the glass was processed, i.e., arrised, ground or polished. At the same time, Sglavo et al. (2007) write that the various edge processing methods are responsible for creating new flaw populations. This is in agreement with Veer (2007), who claims that grinding operations might increase the damage because the experimental data results he obtained indicated that some as-cut specimens were stronger on average. This has been noted by others too (Lindqvist, 2013; Vandebroek et al., 2014). In summary, there is an on-going debate in the literature over the significance of grinding and polishing operations on edge flaw population characteristics. The data in the present study suggests that grinding and polishing operations could be responsible for increasing the average value of $\mathrm{COV}$ for strength.

Figure 4a representing the as-cut edge statistics indicates a possible scale-dependency of the shape parameter. Although such scale-dependency has not been addressed previously in the context of structural glass engineering so far as the authors are aware, it has been noted elsewhere for metal fatigue in components such as gears, shafts and turbine blades, the strength of gear pairs made from plastic and roller bearings (Juskowiak and Bertsche, 2014, see also Seo et al., 2009). The salient feature is that the shape parameter decreases with increasing scale within a Weibull analysis framework. Considering the statistics in Fig. 4, it appears that one effect of grinding and polishing could be to remove a scale-dependency on the shape of the Weibull distribution, although more research is needed to verify this.

Considering the as-cut edge type, for which one side has been scribed and thus subjected to mechanical action (compare Fig. 1A), it is logical to expect the mean strength to increase when the non-scored edge (part 1 in Fig. 1A) is positioned in the tension zone compared to the scored edge (part 2 Fig. 1A) when applying four- point bending with an out-of-plane configuration. This is also reflected in Fig. 4a for the four data samples that represent this type of bending configuration (green marker colour in the figure corresponds to out-of-plane bending configuration with the scored edge positioned in tension zone while red marker corresponds to the nonscored edge subjected to tension). However, what about potential differences in edge strength with an in-plane bending configuration compared to an out-of-plane configuration? Most of the data on the edge strength comes from specimens loaded with an in-plane configuration of bending. There are significant ad- vantages with the inplane compared to the out-of-plane configuration when measurements of edge strength are sought if it is assumed that results from in-plane bending tests can be used as a proxy for edge strength. In contrast, with an out-of-plane configuration, a substantial portion of failures may not occur from the edge, but on the centre part of the surface. Table 4 provides an indication of the ratio of edge to surface failures that may occur in practical circumstances. If the purpose is to obtain a sample of, e.g., 30 observations of edge strength using an out-of-plane bending configuration then according to Table 4 one may in the worst case have to be prepared to put some 150 specimens to the test, although on average about 60 will suffice. There is the additional time and effort involved in identifying each fractured specimen according to whether it failed at the surface or from the edge. More research is needed however, to verify whether the recorded edge strength distribution is the same for in-plane loading as for out-of-plane.

In this study, the influence of the cross-sectional and longitudinal fracture location was generally not considered except in the analysis of the as-cut edge, cf. Fig. 1. The influence of these location descriptors was studied by Vandebroek et al. (2014) who found that in the case of raw-cut edges, a considerably larger number of failures originated from the mechanically scribed edge. The same was found by Kleuderlein et al. (2014). However, according to Vandebroek et al. (2014), the observed strength was "almost not depending" on crosssectional location. Moreover, the edge strength value was "hardly dependent" on longitudinal failure location, although the frequency of failures varied in the longitudinal direction (Vandebroek et al. 2014). This is in general accord with the results reported in Sglavo et al. (2007).

Kleuderlein et al. (2014) obtained their glass specimens from six different suppliers. The process parameters were documented by each manufacturer and the protocols were compared. The results showed that the suppliers used cutting machines from different companies and moreover, used different cutting pressure, cutting speed, cutting wheel angle and cutting fluid, even for the same glass thickness. Kleuderlein et al. (2014) also noted significant differences in how the arrised edge type in particular was produced, e.g. in terms of the 
optical quality. Their results are not entirely conclusive as to the effect on strength due to edge processing type, i.e. cut, arrised, or ground. However, they conclude that different production parameters lead to different strength levels. This is in general agreement with other studies, see e.g. Veer et al. (2006); Le Bourhis (2008); Lindqvist (2013); Vandebroek et al. (2014). According to Veer (2007), the degree of maintenance is more important for the strength than the other settings of the grinding machine. According to EN 16612:2019, the edge strength reduction factor is defined so that when the abrasive action is across the edge, the strength is $11 \%$ lower than when the abrasive action is along the length of edge for arrised and ground processing types. Vandebroek et al. (2014) note that glass which originates from different manufacturers and which is processed at different points in time or in different factories might exhibit varying strength levels due to the presence of residual compressive stresses. Residual stresses develop as a consequence of the annealing process. The pre-stress levels are not very consistent according to Veer et al. (2009) who measured the residual stresses in specimens prior to testing them until failure and they conclude that the production process is less controlled than commonly assumed. All of this suggests that it is relevant to quantify and include in a model the potentially confounding effect due to glass supplier/batch. This was also considered in the present analysis where the supplier was included as a mixed-effect in a linear statistical model. The results show that the incorporation of supplier effects can help to explain a significant part of the total variability in observed Weibull parameter estimates.

\section{Conclusion}

A comprehensive analysis of glass edge strength is performed based on a survey of experimental measurements which can be found in a range of journal articles, conference proceedings and academic dissertations available in the open literature. Tests with four types of glass edge in a four-point bending fixture show that there is substantial variability in Weibull distribution parameter estimates, even when specimens with the same dimensions from the same supplier are subjected to the same loading rate in an in-door climate. Numerical investigations show that when sample size is limited, Weibull shape parameter estimates scatter substantially and this can provide an explanation for some of the observed variability (in shape). Data from some 1800 measurements comprising up to 19 suppliers of glass strongly suggest that there are differences in Weibull shape factor between edge types. The polished edge is associated with the lowest Weibull shape factor on average and the arrised with the highest whereas as-cut and ground edges lie in-between. Additionally, the data indicates that for the as-cut edge, the shape parameter is scale-dependent with an inverse proportionality, i.e., the lower the scale the higher is the shape value. This scaledependency of shape appears to vanish with ground and polished edge types thus indicating a possible effect of grinding operations on the strength distribution.

The variability in parameter estimates can be further explained by considering the parameters as random variables nested within a Weibull random variable. The linear mixed-effects statistical model is used with the supplier as mixed-effect. Predictions made while assuming that glass is obtained from an unknown batch or supplier (i.e., from any of the suppliers in the study) show that the characteristic, 5percentile strength (considering its lower bound in a one-sided confidence interval at the $75 \%$ level) is 42 $\mathrm{MPa}, 45 \mathrm{MPa}, 48 \mathrm{MPa}$ and $46 \mathrm{MPa}$ for the as-cut, arrised, ground and polished edge type, respectively, on a reference $100 \mathrm{~mm}$ load span at $2 \mathrm{MPa} \mathrm{s}^{-1}$ stress rate. The size effect can be represented by the usual weakest-link scaling formula if the Weibull modulus is taken to be $8.0,12.0,8.0,6.5$, for the respective edge-types. The estimated stress corrosion parameter is close to $n=16$ with an approximate $95 \%$ confidence interval $(14.0,16.9)$. The size effect in this study is based on a simplified representation of the edge as a line and thickness is neglected. In reality, glass thickness may interact with edge length and edge type to produce an effect on strength in ways that may not be self-evident, e.g. due to modified machine settings that apply when cutting glass of different thickness. More research is needed to assess the effect on strength due to thickness and how this may interact with edge length and edge profile. The equations used in this study to motivate the chosen linear mixed-effects statistical models are in reality coupled and this was neglected in a simplified approach. In future work this may be addressed and resolved more fully.

The hierarchical modelling approach results in the same distribution shape parameter for all data corresponding to the same edge-type. By considering the data sets in groups according to edge type, it is possible to estimate the Weibull parameters using a clustered likelihood estimator under the condition that the shape value is the same for the same edge type. It is found that the as-cut, arrised, ground and polished edge has a shape value quite close to those estimated with the hierarchical model and a very similar scale value.

\section{Author Contributions}

David Kinsella: Is the main author of the article and the conceptual designer of the project, the main analysis and the interpretation of data.

Johan Lindström: Contributes to the statistical analysis and interpretation of data and to the revision of the manuscript. 


\section{Conflict of Interest}

On behalf of all authors, the corresponding author states that there is no conflict of interest.

\section{References}

Akaike, H., 1969. Fitting autoregressive models for prediction. Annals Institute Stat. Math., 21: 243-247.

Broek, D., 1983. Elementary Engineering Fracture Mechanics. 3rd Edn., Springer Netherlands, ISBN-10: 9401183708, pp: 469.

Brown, W.G., 1974. A Practicable Formulation for the Strength of Glass and its Special Application to Large Plates. 2nd Edn., National Research Council Canada, pp: 62.

Burnham, K.P. and D.R. Anderson, 1998. Model selection and inference.

Calderone, I., 1999. The equivalent wind loading for window glass design. PhD Thesis, Monash University.

Carre, H., 1996. Etude du comportementa la rupture d'un materiau fragile precontraint: Le verre trempe. $\mathrm{PhD}$ Thesis, Ecole Nationale des Pontset Chauss'ees.

Charles, R.J., 1958a. Static fatigue of glass I. J. Applied Phys., 29: 1549-1553.

Charles, R.J., 1958b. Static fatigue of glass II. J. Applied. Phys., 29: 1554-1560.

DIN 18008-1, 2010. Glas im Bauwesen- Bemessungsund Konstruktionsregeln - Teil 1: Begriffe und allgemeine Grundlagen. CEN

EN 1288-3, 2000. Glass in building - Determination of the bending strength of glass - Part 3: Test with specimen supported at two points (four point bending). CEN

EN 16612, 2019. Glass in building - Determination of the lateral load resistance of glass panes by calculation. CEN

EN 572-1, 2012. Glass in building - Basic soda lime silicate glass products - Part 1: Definitions and general physical and mechanical properties. CEN

Evans, A.G., 1974. Slow crack growth in brittle materials under dynamic loading conditions. Int. J. Fracture, 10: 251-259.

Givens, G.H. and J.A. Hoeting, 2013. Computational Statistics. 2nd Edn., John Wiley and Sons, ISBN-10: 0387981454, pp: 728.

Haldimann, M., 2006. Fracture strength of structural glass elements-analytical and numerical model- ling, testing and design. PhD Thesis, Ecole Polytechnique Federale de Lausanne EPFL.

Hellan, K., 1984. Introduction to Fracture Mechanics. 1st Edn., McGraw-Hill, ISBN-10: 0070663505 , pp: 302 .
Irwin, G., 1958. Fracture. In: Handbuch der Physik, Irwin, G. (Ed), Springer, p: 551.

Irwin, G.R., 1957. Analysis of stresses and strains near the end of a crack traversing a plate. J. Applied Mech., 24: 361-361.

Johar, S., 1981. Dynamic fatigue of flat glass-phase technical report, Ontario research foundation, Department of Metals, Glass and Ceramics.

Johar, S., 1982. Dynamic fatigue of flat glass - Phase. Technical report, Ontario Research Foundation, Department of Metals, Glass and Ceramics

Juskowiak, J. and B. Bertsche, 2014. Stress-dependent Weibull shape parameter based on field data. In: Probabilistic Safety Assessment and Management PSAM 12, Juskowiak, J. and B. Bertsche, Honolulu, Hawaii

Kanabolo, D.C. and H.S. Norville, 1985. The strength of new window glass using surface characteristics. MSc Thesis, Texas Tech University.

Kleuderlein, J., F. Ensslen and J. Schneider, 2014. Investigation of edge strength dependent on different types of edge processing. Proceedings of the International Conference at Glasstec Du“ sseldorf Engineered Transparency, (DET' 14). Germany.

Kozlowski, M., 2014. Experimental and numerical analysis of hybrid timber-glass beams. PhD Thesis, Silesian University of Technology.

Krohn, M.H., J.R. Hellmann, D.L. Shelleman and C.D. Pantano, 2002. Biaxial flexure strength and dynamic fatigue of soda-lime-silica float glass. J. Am. Ceram Soc., 85: 1777-1782.

Lawn, B., 1993. Fracture of Brittle Solids. 2nd Edn., Cambridge University Press, ISBN-10: 0521409721, pp: 378.

Le Bourhis, E., 2008. Glass. Wiley-VCH Verlag GmbH and $\mathrm{Co} \mathrm{KGaA}$, Weinheim

Lehmann, E.L. and G. Casella, 1998. Theory of Point Estimation. 1st Edn., Springer Texts in Statistics, ISBN-13: 978-0-387-22728-3.

Lindqvist, M., 2013. Structural glass strength prediction based on edge flaw characterization. PhD Thesis, Ecole Polytechnique F'ed'erale de Lausanne EPFL.

MathWorks, Inc., 2018. www.mathworks.com

McCulloch, C.E., S.R. Searle and J.M. Neuhaus, 2008. Generalized, Linear and Mixed Models. 2nd Edn., Wiley, ISBN-10: 0470073713, pp: 424.

McLellan, G.W. and E.B. Shand, 1984. Glass Engineering Handbook. 3rd Edn., McGraw-Hill, ISBN-10: 007044823X, pp: 471.

Mencik, J., 1992. Strength and fracture of glass and ceramics, glass science and technology.

Mesarovic, S., D. Gasparini, S. Muju and M. McNelis, 1992. Probability of crack growth in Poisson field of penny cracks. J. Eng. Mech., 118: 961-978. 
Müller-Braun, S., M. Seel, M. König, P. Hof and J. Schneider et al., 2020. Cut edge of annealed float glass: crack system and possibilities to in-crease the edge strength by adjusting the cutting process. Glass Struct. Eng., 5: 3-25.

DOI: $10.1007 / \mathrm{s} 40940-019-00108-3$

Muniz-Calvente, M., A. Ramos, F. Pelayo, M.J. Lamela and A. Fernandez-Canteli, 2016. Statistical joint evaluation of fracture results from distinct experimental programs: An application to annealed glass. Theor. Applied Fract. Mec., 85: 149-157.

Natividad, K., S.M. Morse, H.S. Norville, 2016. Fracture origins and maximum principal stresses in rectangular glass lites. J. Archit. Eng., 22:1-1. DOI: $10.1061 /($ ASCE)AE.1943-5568.0000197

Osnes, K., O. Hopperstad and T. Børvik, 2018. Quasistatic and Dynamic testing of annealed float glass. Proceedings of the 18th International Conference on Experimental Mechanics (CEM' 18), Brussels, Belgium.

Rinne, H., 2009. The Weibull Distribution - a Handbook. 1st Edn., Taylor and Francis Group CRC Press, ISBN-10: 1420087444, pp: 808.

Ritter, J.E., P. Strzepa, K. Jakus, L. Rosenfeld and K.J. Buckman, 1984. Erosion damage in glass and alumina. J. Am. Ceram Soc., 67: 769-774.

Sampson, P.D., A.A. Szpiro, L. Sheppard, J. Lindstr"om and J.D. Kaufman, 2011. Pragmatic Estimation of a Spatiotemporal air quality model with irregular monitoring data. Atmospheric Environ., 45: 6593-6606.

Schula, S., 2015. Charakterisierung der Kratzanf'alligkeit von Glasernim Bauwesen. 1st Edn., Springer-Verlag, ISBN-10: 3662477823, pp: 406.

Seo, J., M. Jung and C. Kim, 2009. Design of accelerated life test sampling plans with a nonconstant shape parameter. Eur. J. Oper., 197: 659-666.

Sglavo, V.M., C. Muller and F. Righetti, 2007. Influence of edge finishing on the resistance to thermal stresses of float glass. Glass Performance.

Vandebroek, M., C. Louter, R. Caspeele, F. Ensslen and J. Belis, 2014. Size effect model for the edge strength of glass with cut and ground edge finishing. Eng. Struct., 79: 96-105.

Vandebroek, M., J. Belis, C. Louter, G.V. Tendeloo, 2012. Experimental validation of edge strength model for glass with polished and cut edge finishing. Eng. Fract. Mech., 96: 480-489.

Veer, F.A. and Y.M. Rodichev, 2011. The structural strength of glass: Hidden damage. Strength Mater, 43: 302-315.

Veer, F.A., 2007. The strength of glass, a nontransparent value. HERON, 52: 87-104.

Veer, F.A., C. Louter and F.P. Bos, 2009. The strength of annealed, heat-strengthened and fully tempered float glass. Fatigue Fract. Eng., 32: 18-25.
Veer, F.A., P.C. Louter and T. Romein, 2006. Quality Control and the Strength of Glass. In: Fracture of Nano and Engineering Materials and Structures, Gdoutos, E.E. (Ed), Springer, ISBN-13: 978-1-4020-4972-9.

Weibull, W., T.C. Tsu, R.A. Mugele and F.A. McClintock, 1952. Discussion of A statistical distribution function of wide applicability. ASME J. Applied Mech.

\section{A) Maximum Likelihood Estimation}

The Weibull parameters can be estimated with the maximum likelihood (ML) method (Lehmann and Casella, 1998, Ch. 6.3), as the (numerical) maximum of the log-likelihood function for the two-parameter distribution in Equation (1):

$$
\begin{aligned}
& \log L(k, m ; \sigma)=n_{0} \log m-n_{0} m \log k+\ldots \\
& \ldots+(m-1) \sum_{i=1}^{n_{0}} \log \sigma_{i}-k^{-m} \sum_{i=1}^{n_{0}} \sigma_{i}^{m},
\end{aligned}
$$

where, $n_{0}$ is the sample size. Approximate confidence intervals for the parameters can be computed using asymptotic theory for ML-estimators (Lehmann and Casella, 1998, Ch. 6.3, for details.). In practice the computations are performed by the wblfit-function in MATLAB (Math Works Inc., 2018).

\section{Likelihood Function for Grouped Data}

An alternative to the model in Equation (19) is to assume that the shape parameter is constant for all samples with the same edge type, while still allowing for different scale parameters. Figure 9 for an illustration of this model. The resulting log-likelihood for all samples from the $q^{\text {th }}$ edge-type becomes:

$$
\begin{aligned}
& \log L\left(k_{q} 1, \ldots, k_{q} N_{q}, m_{q} ; \sigma\right)=n_{q} \log m_{q}-\ldots \\
& -\ldots m_{q} \sum_{j=1}^{N_{q}} n_{q j} \log k_{q j}+\ldots \\
& \ldots+\left(m_{q}-1\right) \sum_{j=1}^{N_{q}} \sum_{i=1}^{n_{q j}} \log \sigma_{q j i}-\ldots \\
& -\ldots \sum_{j=1}^{N_{q}} k_{q j}^{-m_{q}} \sum_{i=1}^{n_{q j}} \sigma_{q j i}^{m q} .
\end{aligned}
$$

Here $m q$ is the shape parameter of the $q^{\text {th }}$ edge-type, $k_{q j}$ is the $j^{\text {th }}$ sample for that edge-type, $n_{q j}$ are the number of observation in the $j^{\text {th }}$ sample, $n_{q}=j n_{q j}$ is the total number of observations for the $q^{\text {th }}$ edge-type and $\sigma_{q j i}$ are the observations (indexed by edge-type, sample and observation number). 


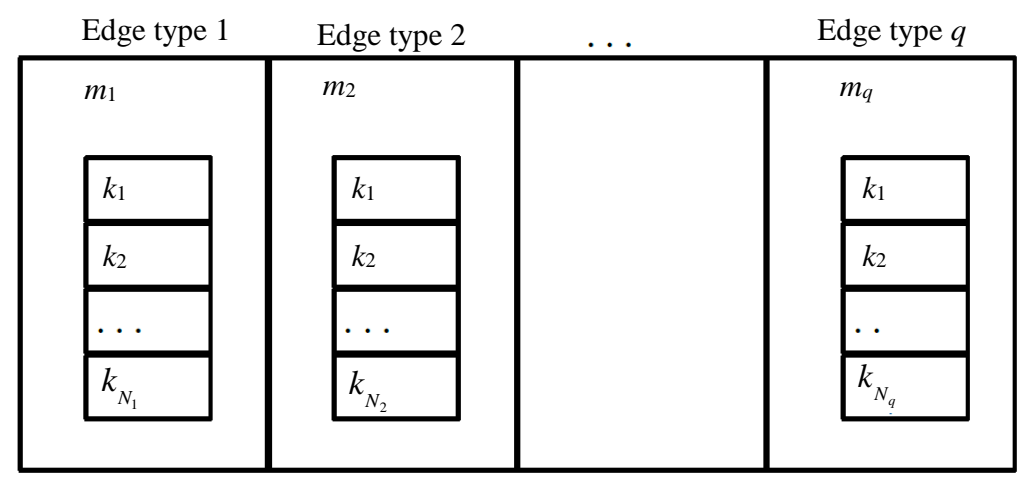

Fig. 9: Model assuming different scale parameters for each sample but a fixed shape parameter for each edge type

To obtain ML-estimates of the parameters in Equation (20) we use the fminunc nonlinear minimization algorithm in MATLAB. The numerical optimization is aided by the computation of first and second derivatives of Equation (20). For a given $q$ and ignoring the $q$ subscripts to simplify notation, we have first:

$$
\begin{aligned}
& \frac{\partial \log L}{\partial k_{j}}=\frac{m}{k_{j}^{m+1}} \sum_{i=1}^{n_{j}} \sigma_{j i}^{m}-\frac{m n_{j}}{k_{j}}, \\
& \frac{\partial \log L}{\partial m}=\frac{n}{m} \sum_{j=1}^{N} n_{j} \log k_{j}+\sum_{j=1}^{N} \sum_{i=1}^{n_{j}} \log \sigma_{j i}+\ldots \\
& \ldots+\sum_{j=1}^{N} \frac{1}{k_{j}^{m}} \sum_{i=1}^{n_{j}} \sigma_{j i}^{m} \log \frac{k_{j}}{\sigma_{j i}},
\end{aligned}
$$

and second derivatives:

$$
\begin{aligned}
& \frac{\partial^{2} \log L}{\partial k_{j} \partial k l}= \begin{cases}-\frac{m(m+1)}{k_{j}^{m+2}} \sum_{i=1}^{n_{j}} \sigma_{j i}^{m}+\frac{m n_{j}}{k_{j}^{2}}, & \text { if } l=j, \\
0, & \text { if } l \neq j,\end{cases} \\
& \frac{\partial^{2} \log L}{\partial m \partial k_{j}}=\frac{1}{k_{j}^{m+1}}\left(\left(1-m \log k_{j}\right) \sum_{i=1}^{n_{j}} \sigma_{j i}^{m}+\ldots\right. \\
& \left.\ldots+m \sum_{i=1}^{n_{j}} \sigma_{j i}^{m} \log \sigma_{j i}\right)-\frac{n_{j}}{k_{j}}, \\
& \frac{\partial^{2} \log L}{\partial m^{2}}=-\frac{n}{m^{2}}+\sum_{j=1}^{N} \frac{1}{k_{j}^{m}} \sum_{i=1}^{n_{j}} \sigma_{j i}^{m}\left(\log \frac{k_{j}}{\sigma_{j i}}\right)^{2} .
\end{aligned}
$$

\section{Model Comparison}

For likelihood based parameter estimation different models can be compared using information criteria, such as the Akaike information criterion (Akaike, 1969), with smaller values indicating better models. The AIC for a model is given by:

$A I C=2 k-2 \log (L)$,

where, $k$ is the number of unknown (or estimated) parameters and $\log (\hat{L})$ is the maximum-value of the log-likelihood. The AIC provides a trade-off between better models (higher value of $\hat{L}$ ) and increasing model complexity (more parameters, $k$ ). When comparing models those within 2 units are considered equivalent, 3-7 units indicates some differences and more than 10 units of difference is seen as strong evidence against the model with larger AIC (Burnham and Anderson 1998, pp. 75-117).

\section{B) Linear Mixed Effects Statistical Model}

Linear Mixed Effects (LME) models are used to identify the source of variation and correlation that arise from clustered data, e.g., when data-collection is undertaken in a hierarchical manner where observational units are related, violating assumptions of independence (McCulloch et al., 2008, Ch. 6). Considering Equation (15b):

$$
\log m_{i}=\sum_{l=1}^{4} \lambda_{l} \mathbb{I}_{\text {edgetype }=l, i}+\sum_{l=1}^{17} c_{l} \mathbb{I}_{\text {supplier }=l, i}+\varepsilon_{i}
$$

this constitutes a LME model where we see the effect of edge-type, $\gamma_{l}$, as fixed regression coefficients but assume that both the supplier (or batch) effect, $c_{l}$ and the residuals, $\varepsilon_{i}$, are samples from normal distributions:

$c_{l} \sim N\left(0, \sigma_{c}^{2}\right)$, and $\varepsilon_{i} \sim N\left(0, \sigma_{\varepsilon}^{2}\right)$.

The suppliers/batch are seen as random effects since: (1) we could expect substantially similar behaviour for glass from the same batch, violating the independence 
assumptions regarding residuals in a standard regression. (2) It is unlikely that the list of suppliers considered here is exhaustive, i.e., that we include all suppliers on the market. (3) Due to the large number of supplier and batches it is more realistic (and useful) to characterise the variability between suppliers/batches than to try to form a complete list of supplier effects. The relative values of the estimated variances in Equation (24) will indicate how much of the variability in shape and scale parameters is due to supplier and how much has to be considered random errors not captured by the regression models in Equation (15).
When predicting from the LME the division in fixed and random effects allows us to consider both the case of a known edge-type and supplier, or the case of a known edge-type but unknown supplier. In both cases standard predictions for the LME will produce expected values for shape and scale parameters, as well as associated uncertainties in these predictions. The uncertainties will be based on the Normal models in Equation (24), with variances estimated from the data. 